\title{
Formation des enseignants en service à intégrer les compétences de la citoyenneté numérique à la lueur de l'approche interdisciplinaire
}

\author{
Nesrine Salah Abdel Ghany
}

Maître de Conférences de la Didactique de FLE

Faculté des Jeunes Filles-Université Ain Shams

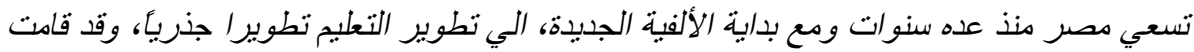



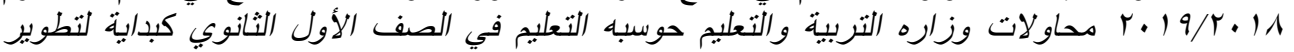
مرحله الثانوية العامة. مانو

وبها ان الطريق قد مهد الان لاستخدام التكنولوجيا وقد لمسه الجميع في كافة محافظات الجمهورية،

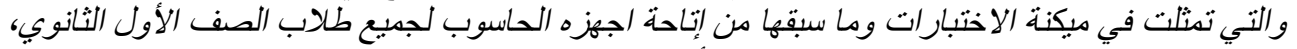

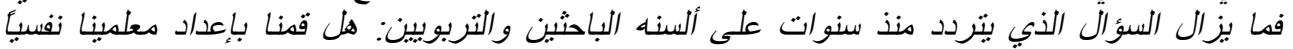

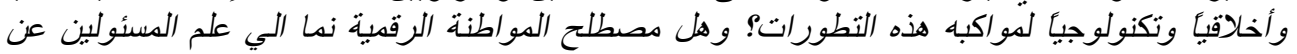



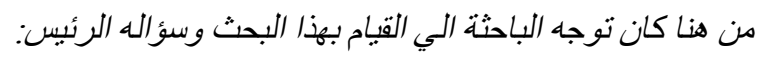

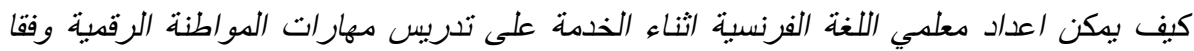
للمدخل البينـي؟



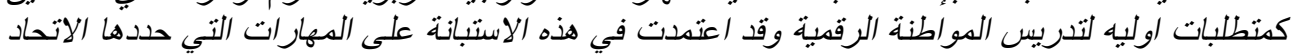

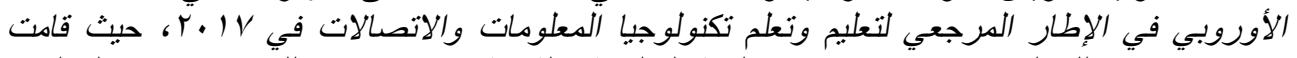


والطلاب وكذلك المؤسسات التعليمبية.

وقد قامت بعد ذلك بإعداد قائمه بههارات المواطنة الرقمية التي يجب علي المعلم اكتسابها ومن ثم

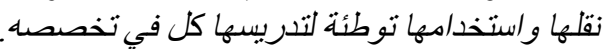

وبعد الاطلاع على برامج تعليم وتعلم المواطنة الرقمية في أوروبا وكندا، قامت الباحثة بإعداد





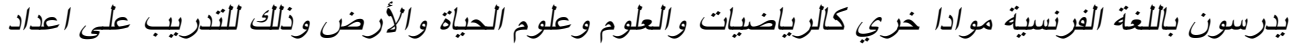
مجموعه من الدروس كل في مجاله.

وقد قامت الباحثة بتطبيق البحث على 11 المعلما من معلدي المدارس الدولية والفرنسية والإنجليزية

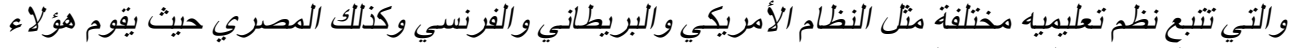

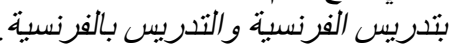
وقد اثبتت نتاح البحث بعد معالجتها إحصائيا فعالية الموديول المقترح 


\section{Introduction :}

Avec le début du millenium, l'information et le savoir ont pris une importance grandissante dans toutes les sociétés modernes. La nécessité de former une population active pourvue de compétences en matière de technologie de l'information et de la communication TIC s'accroit et l'utilisation des nouvelles technologies dans l'éducation implique de nouveaux rôles pour les enseignants, de nouvelles pédagogies, de nouvelles approches de formation des enseignants.

Aussi l'un des défis principaux du $21^{\mathrm{e}}$ siècle est le numérique: puisque les données ne sont pas les informations, et les informations ne font pas la connaissance et la connaissance ne fait pas la manière de décider quelque chose, alors l'apprenant doit apprendre le numérique, et surtout apprendre à apprendre avec le numérique. Le rôle d'accompagnement des enseignants, est de prendre la responsabilité de guider les élèves à passer des données à l'information, de l'information à la connaissance, de la connaissance au savoir-faire dans la vie. Par conséquent, les enseignants doivent être très bien formés, pour qu'ils soient non seulement en mesure de moduler un numérique d'urgence permanente, mais aussi de savoir ralentir afin de mieux réfléchir plus correctement. (Lumbrozo, O. 2017.)

Dans tout pays développé ou en développement, il est admis que les technologies sont au centre de la réflexion mondiale et s'insèrent dans tous les domaines. Le rythme de l'innovation dans les technologies de l'information et de la communication numérique s'accélère: "Opter pour les Nouvelles technologies ", c'est se préparer pour affronter la société du savoir. (Perrenoud. Ph, 2011

Conscient de ces faits, le gouvernement égyptien se dit prêt à agir pour favoriser l'intégration des technologies numériques et ainsi améliorer les compétences de la main d'œuvre, promouvoir l'efficacité de l'école, renforcer la qualité de l'éducation et de l'apprentissage, permettre aux enseignants de répondre aux exigences professionnelles, etc. C'est d'ailleurs une vision que le gouvernement voulait incarner depuis des années dans les institutions publiques, les écoles, le marché du travail, etc.

En effet, la formation du personnel et des enseignants devient une réalité efficace pour une intégration réussie de la technologie en Égypte. D'ailleurs, les responsables avaient même 
entrepris la formation des cadres éducatifs sur l'intégration des TIC en enseignement pour qu'à leur tour, ils la transmettent aux enseignants et au personnel œuvrant dans le système scolaire.

La formation professionnelle des enseignants est considérée comme un volet essentiel de l'amélioration de l'éducation surtout à l'ère numérique, de même l'intégration des technologies de l'information et du communication et de l'enseignement (TICE) exige de l'enseignant des aptitudes nouvelles; dans cette perspective, l'UNESCO et ses partenaires, CISCO, INTEL, ont publié un référentiel (TIC UNESCO 2012) de compétences TIC pour les enseignants, dans le but de définir les compétences requises d'un enseignement efficace qui aident à préparer un citoyen doté par l'esprit créatif, capable de traiter les informations numériques et de disposer de connaissances et des ressources permettant à gérer efficacement leur propre vie, et de mener une existence riche et satisfaisante.

En 2018, ils ont publié une nouvelle version destinée à informer les responsables de l'élaboration des politiques éducatives, les formateurs des enseignants et les fournisseurs de formation professionnelle et les enseignants en activité du rôle que jouent les TIC dans la réforme de l'éducation.

$\mathrm{Ce}$ référentiel répond à la nécessité de l'intégration des TICE en classe, qui sera plus ou moins réussie selon la capacité dont fera preuve l'enseignant pour structurer l'environnement d'apprentissage de façon innovante, fusionner les nouvelles technologies avec de nouvelles pédagogies et créer une classe socialement active, en stimulant l'interaction coopérative, l'apprentissage collaboratif et le travail de groupe.

Ce Référentiel est basé sur trois étapes successives de la formation de l'enseignant « l'Alphabétisation

technologique au sens que les apprenants doivent utiliser les TIC en vue d'un apprentissage plus efficace, et l'Approfondissement des connaissances qui vise à l'acquisition des connaissances approfondies dans les disciplines étudiées à l'école afin d'aider les enseignants à résoudre des problèmes complexes et concrets dans la vie. Et finalement la Création de connaissances : selon cette étape, les apprenants, considérés comme étant des citoyens et des futurs acteurs de l'économie, doivent créer les nouveaux savoirs indispensables à rendre les sociétés plus harmonieuses et prospères. 
Ce Référentiel plaide pour que les enseignants utilisent des méthodes d'enseignement adaptées à des sociétés du savoir en constante évolution, en vue d'aider leurs apprenants, non seulement à acquérir une connaissance approfondie des disciplines qu'ils étudient, mais aussi à comprendre comment eux-mêmes peuvent générer de nouveaux savoirs en se servant des TIC.

Parallèlement à la parution de ce référentiel, l'Égypte aussi a commencé ses premiers pas vers l'intégration numérique dans l'enseignement. Le gouvernement égyptien a débuté par l'accessibilité du tablette pour la plupart des apprenants (pour la première année secondaire de l'année scolaire 2018/2019); ainsi le gouvernement a transformé l'évaluation de cette même promotion en numérique; mais un regard auprès du statut actuel de nos enseignants mène à poser une question indispensable: sont-ils prêts à cette révolution, sont-ils dotés des compétences numériques qui leurs permettent d'intégrer et de manipuler les TICE dans leur enseignement, d'ici s'émerge la naissance de cette recherche.

Afin d'évaluer le niveau de nos enseignants, la chercheuse s'est appuyée sur le référentiel du UNISCO pour repérer les compétences TICE qui correspondent avec

l'environnement et la nature de l'enseignement égyptien, le niveau technologique des écoles avec ses diversités, et qui s'adaptent aussi avec le niveau technologique des enseignants.

Mais est ce que le problème réside dans l'insuffisance des compétences technologiques ? Bien sûr que non, le fait d'être né à l'époque du numérique ne signifie pas forcément que l'on maitrise le numérique ; surtout qu'il existe un risque qu'un fossé se creuse entre ceux qui accroissent leurs compétences pour récolter les fruits de leur utilisation des nouvelles technologies et en éviter les écueils et ceux qui abandonnent et se retrouvent victimes du (cyber-harcèlement, des tentatives d'hameçonnage, et plus récemment de la radicalisation en ligne.) (Matthew J. 2012)

Pour toutes ces raisons, les pédagogues ont commencé à intégrer le terme de l'éducation à la citoyenneté numérique, un nouveau domaine de l'éducation, qui a paru pour lutter contre les problèmes déjà mentionnés et qui vise aussi à rendre les apprenants plus autonomes, pour pouvoir faire des choix informés quand ils font face au potentiel qu'offrent les nouvelles technologies.

Les recherches dans ce domaine semblent être rares. On 
peut citer celle de (Shaaban S. et El Sayed M. 2014), qui ont déterminé les 9 composantes de la citoyenneté numérique : (l'accessibilité numérique, le commerce numérique, la communication numérique, l'alphabétisation numérique, les lois numériques, les droits et les responsabilités numériques, la santé et la sécurité numérique). Ils ont aussi présenté les étapes du développement de la citoyenneté numérique qui se résume dans la conscience de ces compétences, la pratique, la modélisation et le bon exemple, l'analyse et le feedback.

Ils ont suggéré quelques modèles pratiques pour l'intégration de la citoyenneté numérique dans des cursus telles que les matières sociales, et ils ont recommandé d'intégrer l'éducation à la citoyenneté numérique dans les stages de formation professionnelles des enseignants de toutes les disciplines.

Dans son étude, (MADEL J. 2014) a déterminé le rôle des enseignants à l'ère du numérique, l'objectif en est une formation continue des enseignants pour un usage adéquat des outils technologiques à travers une communauté de pratique en réseau social. La formule sera d'en appeler aux ressources des participants et aussi de chercher des ressources extérieures pour le renforcement des capacités pédagogiques et technologiques.

Alors la rareté des études surtout dans le domaine de la didactique de la langue française en Égypte; et l'orientation du gouvernement vers le numérique, toutes ces éléments encouragent tout chercheur à étudier l'intégration de l'éducation à la citoyenneté numérique dans son domaine.

Mais avant de commencer à intégrer l'éducation à la citoyenneté numérique dans nos programmes de formation, il est nécessaire d'analyser l'état actuel des enseignants, des écoles, de l'environnement technologique de notre système éducatif. Ce qui a mené la chercheuse à accorder des rencontres avec 18 enseignants de diverses écoles (religieuses, internationales, d'investissements anglophone et francophone), qui enseignent selon divers systèmes éducatifs (égyptien, français, américain et anglais). Les résultats de ces rencontres se résument ainsi :

- La plupart des écoles ont des accès Internet et elles sont bien équipées par les moyens qui mènent à l'intégration de la technologie.

- Le manque crucial des valeurs et des attitudes modérant l'usage numérique est un défi 
qui affectent le comportement de leurs élèves.

- L'éducation à la citoyenneté numérique est un nouveau terme pour eux ; ils souhaitent être y bien formé pour pouvoir intégrer les compétences et les valeurs de la citoyenneté numérique dans leurs disciplines.

- Le besoin de coopération entre les différentes disciplines pour intégrer la citoyenneté numérique semblent être plus efficace pour enseigner les valeurs et les attitudes de ce domaine récent.

Avant de procéder dans cette recherche, une autre question semble être primordiale : est-ce que le contenu de l'éducation à la citoyenneté numérique se place parmi les contenus transversaux ? Reverdy, C. (2015) répond : "L'engouement pour les contenus transversaux n'est pas nouveau à l'école, puisque, les finalités éducatives n'ont jamais été uniquement centrées sur la seule transmission de savoirs, mais au contraire sur la formation des esprits des élèves à l'éducation à la citoyenneté numérique, leur faire acquérir de nouvelles responsabilités dans un monde complexe. L'école doit ainsi, par la formation de futurs citoyens d'une société donnée, répondre à la demande sociétale en faisant évoluer ses matières scolaires ou en intégrant des contenus transversaux dans les curriculums ». C'est exactement ce que cette recherche actuelle vise réaliser.

La citoyenneté numérique se place parmi les contenus transversaux qu'on peut enseigner selon l'approche interdisciplinaire, comme l'éducation à la démocratie, à la santé et aux médias, de plus en plus, on considère que "l'éducation n'a de valeur que dans la mesure où elle rencontre les besoins des apprenants"; Reverdy, C. (2015) On réalise que si l'éducation veut avoir un sens, elle doit aider les apprenants à trouver des réponses à des problèmes de vie réelle qui les concernent: "l'éducation doit préparer à la vie ».

La vie et ses problèmes actuels ne sont pas cloisonnés en disciplines et doivent être abordés par des démarches interdisciplinaires car, "un argument essentiel en faveur de l'interdisciplinarité est que les disciplines ne donnent pas et ne donneront jamais une image complète des choses si on les considère isolément. C'est en s'articulant entre elles, en s'intégrant, qu'elles jouent le rôle les plus efficace". En outre, "percevoir l'unité et la diversité ou la spécificité des choses représente 
une finalité de l'apprentissage, qui correspond à un besoin de l'apprenant et à une réalité à découvrir". Reverdy, C (2015)

Les recherches en éducation montrent que les enseignants éprouvent de manière générale des difficultés à mettre en œuvre une réelle interdisciplinarité dans leurs classes et les pratiques qui en découlent relèvent plus souvent d'une pluridisciplinarité, c'est-àdire une juxtaposition de matières qui restent cloisonnées malgré un objectif commun.

Plusieurs facteurs sont à l'origine de cette difficulté : la formation des enseignants du secondaire, très disciplinaire et peu tournée vers l'épistémologie, qui s'intéresse justement à la spécificité, l'histoire et la philosophie des disciplines; la forme scolaire et les instructions officielles qui, ne favorisent ni une réelle évaluation interdisciplinaire, ni le débat, ni le développement de l'esprit critique ou de l'engagement des élèves.

Reverdy, C. affirme que les enseignants puissent encourager l'engagement des apprenants à prendre part aux questions vives de leur société; cela nécessite un accompagnement global des acteurs de l'établissement, et à offrir aux apprenants des occasions de s'engager concrètement.
Alors quelles compétences et quelles connaissances travailler en priorité dans le cadre de la formation des enseignants par le biais de l'interdisciplinarité ? De quelle manière? et en vue de quelle finalité ?

Tous ces faits ont encouragé la chercheuse à aborder ce nouveau domaine afin de développer les compétences des enseignants et leur aider à intégrer les valeurs et les attitudes de la citoyenneté numérique selon une approche interdisciplinaire: sujet de cette recherche.

\section{Problématique de la recherche :}

La problématique de la recherche réside dans la question suivante : " comment former les enseignants en service à intégrer les compétences de la citoyenneté numérique à la lueur de l'approche interdisciplinaire afin de les préparer à l'ère numérique.

De cette question émanent les questions suivantes :

- Quelles sont les compétences de la citoyenneté numérique censées acquises par les enseignants en service?

- Jusqu'à quel point les enseignants sont dotés de ces compétences de la citoyenneté numérique?

- Quel est le module de formation proposé basé sur l'approche interdisciplinaire 
pour aider les enseignants en service à intégrer les compétences de l'éducation à la citoyenneté numérique ?

- Quel est l'efficacité du module de formation proposé basé sur l'approche interdisciplinaire pour aider les enseignants en service à intégrer des compétences de l'éducation à la citoyenneté numérique?

Les objectifs de la recherche: Cette recherche vise à :

- Déterminer les compétences de la citoyenneté numérique censées acquises par les enseignants en service?

- Préparer un module de formation basé sur l'approche interdisciplinaire pour aider les enseignants en service à intégrer les compétences de l'éducation à la citoyenneté numérique

- Aider les enseignants en service à intégrer les compétences de la citoyenneté numérique dans leurs classes

- Développer les compétences de l'enseignement de la citoyenneté numérique des enseignants en service

\section{Méthodologie de la recherche:}

La chercheuse a suit la méthode mixte entre la méthode descriptive et quasiexpérimentale dans l'application de la recherche.

\section{Délimites de la recherche :}

- L'échantillon de la recherche se compose de 18 enseignants de français et de DNL (discipline non linguistique), enseignant de mathématique et de sciences, dans des écoles francophones au Caire, il dispose d'un niveau linguistique (B1) .

- La Place de l'exécution de la recherche était une salle à l'école des «Frères 》 à Babellouq au centre du Caire

- Le temps proposé pour exécuter cette recherche est 20 heures à raison de deux heures par semaine .

\section{Hypothèses de la recherche}

- Il existe une différence significative statiquement entre la moyenne des notes du groupe expérimental au pré/post-test des compétences de la citoyenneté numérique, en faveur du post-test (en tout)

- Il existe une différence significative statiquement entre la moyenne des notes du groupe expérimental en ce qui concerne les valeurs, les attitudes et les compétences de citoyenneté numérique (au pré/post-test en faveur du posttest

- Il existe une différence significative statiquement entre la moyenne des notes du 
groupe expérimental en ce qui concerne la préparation d'une leçon de la citoyenneté numérique au pré / post-test en faveur du post-test.

Procédure de la recherche : La chercheuse suit les étapes suivantes :

- Une vue approfondie des études et des recherches concernant la citoyenneté numérique s'avère indispensable, ce qui aidera la chercheuse à déterminer les compétences de la citoyenneté numérique valables au niveau des enseignants afin de pouvoir les intégrer; chacun selon sa discipline, tout en se basant sur l'approche interdisciplinaire.

- Élaborer un questionnaire concernant les compétences de la citoyenneté numérique en guise de déterminer le niveau d'acquisition de ces compétences ; elle les a sélectionnées parmi les compétences de la citoyenneté numérique citées par le conseil de l'Europe en 2017

- Élaborer un pré/ post-test des compétences de la citoyenneté numérique pour déterminer jusqu'à quel point les enseignants en service sont dotés des compétences concernant l'éducation à la citoyenneté numérique.

- Préparer le module de formation proposé, en débutant par une étude approfondie de l'approche interdisciplinaire qui représente le cadre philosophique de la recherche visée; tout en présentant les programmes de formation des citoyens numériques menées par le conseil d'Europe ; ce qui a aidé la chercheuse à déterminer les objectifs du module, le contenu, les modalités de travail et les modes d'évaluation.

- Préparer une leçon selon l'approche interdisciplinaire afin d'intégrer une séance concernant la citoyenneté numérique.

- Utiliser une_orille d'observation pour évaluer les performances de l'éducation de la citoyenneté numérique des enseignants en service.

- Appliquer une fiche d'autoévaluation afin de mesurer le degré d'acquisition des valeurs et des compétences concernant la citoyenneté numérique des enseignants en service.

- Présenter un portfolio professionnel qui reflète leurs expériences et leurs 
commentaires

durant

l'application de la recherche.

- Ré-application du pré/post-test pour mesurer l'efficacité du module de formation sur l'éducation à la citoyenneté numérique des enseignants en service.

Importance de la recherche : $\mathrm{La}$ présente recherche tend à :

- Aider les enseignants en service à apprendre $d u$ numérique, et aussi apprendre à apprendre avec le numérique.

- Aider leurs apprenants à être de bons citoyens numériques.

- Mettre en valeur l'importance de l'éducation à la citoyenneté numérique, domaine jusqu'à présent ignoré des programmes de la formation des enseignants égyptien malgré son importance.

- S'orienter aux modules de formation des enseignants introduisant la citoyenneté numérique

- Mettre à la disposition des enseignants, des outils utiles dont ils peuvent utiliser pour intégrer l'éducation à la citoyenneté numérique dans leurs disciplines.

- Envisager des pistes pédagogiques variées, flexibles et applicables pour l'enseignement des compétences et des valeurs de la citoyenneté numérique selon l'approche interdisciplinaire.

- S'orienter vers les projets interdisciplinaires dans leur vie professionnelle.

- Prendre la responsabilité de guider leurs apprenants à passer des données de l'information à la connaissance, et de la connaissance au savoir-faire dans la vie.

Échantillon de la recherche:

L'échantillon de la recherche est hétérogène, il se compose de 18 enseignants de français et de discipline non linguistique dans des écoles religieuses, des écoles d'investissements :

(francophones et anglophones) et des écoles internationales. L'expérience de ces enseignants varie de 5 à 15 ans avec les cycles primaires, préparatoires et secondaires, ils enseignent selon des différents systèmes éducatifs, tels que le système égyptien, le bac français, le système britannique (IGCSE), et le système américain.

Outils de la recherche: Afin de préparer ce module de formation proposé, la chercheuse a élaboré les outils et les matériels suivants :

- Un questionnaire ayant pour but de déterminer les 
compétences

technopédagogiques

préalables à l'acquisition de la citoyenneté numérique des enseignants en service.

- Une grille des compétences de la citoyenneté numérique nécessaires pour préparer un citoyen numérique selon les critères, les coutumes et les données de notre société.

- Un pré/post test pour mesurer l'efficacité du module de formation proposé.

- Une fiche de préparation pédagogique des activités pour intégrer la citoyenneté numérique selon l'approche interdisciplinaire.

- Une grille d'observation pour évaluer les performances des enseignants en service.

- Une fiche d'auto-évaluation qui aident les enseignants en service de s'autoévaluer en ce qui concerne leurs compétences de la citoyenneté numérique.

- Un portfolio professionnel pour noter les commentaires, les observations et les avis des enseignants pendant et après l'expérimentation de la recherche.

\section{Terminologies de la recherche:}

- La citovenneté numérique: C'est le maniement efficace et positif des technologies numériques (créer, travailler, partager, établir des relations sociales, rechercher, jouer, communiquer et apprendre) et c'est la participation active et responsable (valeurs, aptitudes, attitudes, connaissance) aux communautés (locales, nationales, mondiales) à tous les niveaux (politique, économique, social, culturel et interculturel). C'est l'engagement dans un double processus d'apprentissage tout au long de la vie (dans des structures formelles, informelles et non formelles) finalement c'est la défense continue de la dignité humaine.

\section{- L'interdisciplinarité :}

Bachelard 1967 signale que l'interdisciplinarité : «C'est la coopération de disciplines diverses, qui contribuent à une réalisation commune et qui, par leur association, permettent l'émergence et le progrès de nouveaux savoir $»$.

Yves Lenoir 2015 affirme que l'interdisciplinarité scolaire c'est la mise en relation de plusieurs matières scolaires qui conduit à l'établissement de liens de complémentarité ou de coopération entre elles, en vue de favoriser l'intégration des 
apprentissages et des savoirs chez les élèves.

Pour la chercheuse, l'interdisciplinarité peut être comprise comme l'association d'éléments de plusieurs disciplines afin d'étudier ou de produire un nouveau savoir.

\section{Cadre théorique :}

On y présente dans les lignes à venir le cadre théorique qui se divise en deux axes principaux: l'interdisciplinarité et la citoyenneté numérique.

Nicole Allieu, M. (1998) a traité l'interdisciplinarité comme étant "l'interaction de deux ou plusieurs disciplines pouvant aller de la communication des idées jusqu'à l'intégration des concepts, des terminologies ou des méthodes».

Partoune, C.(1996) signale que : "L'interdisciplinarité, c'est travailler ensemble pour reconstruire une réalité morcelée artificiellement par le cloisonnement des disciplines et viser l'acquisition de compétences transversales. Il ne s'agit pas de rechercher l'interdisciplinarité parce que c'est une valeur en soi. C'est une approche qui s'impose dès lors qu'on aborde un problème concret, un projet, un sujet, ou un concept.

Vaideanu,

considère que l'interdisciplinarité est d'autant mieux atteinte que les disciplines sont plus intégrées et qu'une discipline ne domine pas une autre dans le processus d'intégration. Il a déterminé quatre catégories dont les caractéristiques sont résumées de la manière suivante :

L'interdisciplinarité de disciplines voisines : c'est le cas dans lequel deux domaines scientifiques deviennent si étroitement imbriqués qu'il y a une zone de chevauchement où les deux disciplines peuvent faire intervenir les méthodes et les concepts qui leur sont propres.

\section{L'interdisciplinarité des} problèmes: $\mathrm{Ce}$ type pourrait s'appliquer à certains groupes de problèmes qui ne peuvent s'inscrire dans le champ d'une discipline déterminée et dont les dimensions sont si nombreuses. La collaboration de plusieurs disciplines devient donc de toute évidence souhaitable.

L'interdisciplinarité des concepts : $\quad \mathrm{Ce}$ type d'interdisciplinarité s'applique aux situations dans lesquelles des modèles et des concepts élaborés à l'intérieur d'une discipline sont ensuite appliqués pour compléter la recherche dans une autre discipline ou même remplacer des modèles et des concepts existants.

Le choix des projets devrait être subordonné à des descriptifs 
détaillés cernant les concepts en cause et indiquant les raisons pour lesquelles ils sont intéressants et peuvent être transférés.

Les définitions de l'interdisciplinarité qu'on vient de citer sont de nature épistémologique et concernent essentiellement l'organisation du savoir, de la recherche et de la résolution de problèmes. Ces conceptions de l'interdisciplinarité ne sont pas essentiellement orientées vers les problèmes d'enseignement et si elles concernent ces problèmes, c'est parce que l'enseignement est intimement lié à la découverte et à l'organisation du savoir.

Pour la recherche actuelle, la chercheuse s'intéresse aux savoirs concernant la citoyenneté numérique, aussi bien ses compétences, ces valeurs et ces attitudes.

Les conditions de réussite du travail interdisciplinaire :

Afin de réussir dans un travail interdisciplinaire il faut :

Avoir des objectifs partagés :_il est important de s'accorder sur des objectifs communs qui doivent être, formulés de manière à faire appel à toutes les disciplines; ils ont pour but de faire converger le travail de l'ensemble de l'équipe et d'orienter les contributions individuelles.

Utiliser au mieux les activités individuelles et collectives : Le travail interdisciplinaire ne signifie pas la participation de chaque membre de l'équipe à toutes les activités. Il est vital d'identifier les contributions de chaque discipline, les compétences individuelles, et par conséquent de programmer et de répartir les tâches.

Comprendre le système de valeurs des autres disciplines : Chaque discipline correspond en fait à un « modèle conceptuel » spécifique. Les membres d'équipe appartenant à plusieurs disciplines auront des savoirs et des valeurs différents. La compréhension de son propre " modèle conceptuel » et de ceux des autres disciplines est nécessaire au travail en commun.

\section{Suivre} un

modèle interdisciplinaire cohérent : Un modèle interdisciplinaire ne doit pas être perçu comme une suite rigide d'étapes, applicable en toute situation. Il s'agit au contraire d'un modèle élaboré par l'équipe en fonction des besoins de l'étude. La construction du modèle est un processus qui ne doit pas naître des outils d'analyse, mais de la confrontation des apports disciplinaires et des intérêts des acteurs autour de la problématique. Elle est issue de la formulation de 
la problématique et des questions de recherche.

A partir de là, l'équipe va pouvoir identifier : ses besoins en information; les concepts les mieux adaptés à l'analyse du problème; les outils les mieux adaptés à la collecte d'information spécifique; la contribution de spécialistes de chaque discipline dans la collecte et l'interprétation de l'information et finalement la procédure spécifique apte à impliquer les groupes d'intérêt dans la démarche (participation directe dans la collecte des données et l'analyse de l'information et de la formulation de propositions ou simple validation des propositions de l'équipe.)

Les principes de l'approche interdisciplinaire : Partoune $\mathrm{C}$. (1996) affirme qu'on doit adopter les idées suivantes pour enseigner selon l'approche interdisciplinaire

- Accepter de plonger dans un sujet inconnu et de continuer, même si l'insécurité déstabilise parfois.

- Accepter de ne pas maîtriser tout le contenu aux yeux des élèves.

- Ne pas se laisser enfermer par la répartition horaire entre les disciplines ; pouvoir imaginer un partage de l'encadrement davantage centré sur la vie du projet plutôt que sur les disciplines

- Être ouvert à l'imprévu et prêt à s'engager dans des apprentissages en fonction des besoins ou des demandes des élèves.

- Doser ses interventions en tant qu'éveilleur à de nouvelles dimensions pour éviter qu'elles deviennent des contraintes, ne répondant pas à une question que se posent les élèves.

- L'enseignant joue le rôle d'homme-orchestre, cela suppose une grande ouverture et une recherche personnelle importante, approfondie, dans d'autres domaines que le sien, afin d'en capter l'essence.

- Une des clés du succès d'une collaboration interdisciplinaire est la convergence suffisante des valeurs et des conceptions de la pédagogie, et une volonté de s'interroger ensemble sur la cohérence de ses pratiques pédagogiques.

- Pour qu'une équipe soit enrichie par la présence d'enseignants de plusieurs disciplines, il faut que chacun d'eux soit suffisamment conscient de ce qu'il peut apporter de spécifique.

- Apprendre à partager ses savoirs, accepter que les 
enseignants mettent en œuvre des séquences qui ne sont pas propres à leurs disciplines, afin de mieux gérer le projet.

- A un moment donné de la conception du module pédagogique, se demander si le regard spécifique dont on est porteur est présent.

La chercheuse a profité de ces principes de l'approche interdisciplinaire dans l'élaboration des outils et des matériels de la recherche.

\section{Obstacles à l'interdisciplinarité}

Perrenoud Ph. (1996) fait le point sur les résistances au travail en équipe, tant pour les institutions que pour les personnes. : «Inutile d'énumérer les difficultés bien connues liées à tout fonctionnement collectif, source de tensions et de conflits, par essence grand consommateur de temps et d'énergie. Le travail en équipe ne devient vraiment un "plus" que dès le moment où ses membres ont acquis un certain nombre de compétences collectives. »

Du côté des établissements, il relève les résistances aux changements structurels (attributions, horaires, organisation matérielle) et la menace de déstabilisation de l'ordre établi, qui repose sur la complicité de la direction, des parents, des autres enseignants et même des élèves, quoi qu'ils en disent.
En ce qui concerne les enseignants, si le groupe des enseignants s'engage dans une gestion collective de la classe, cela suppose de dévoiler le cadre de référence de chacun en ce qui concerne les règles de vie et les pratiques méthodologiques et d'arriver à se mettre d'accord sur un cadre commun. Cela exige que chacun puisse justifier ses manies ou ses exigences, ou alors les laisse tomber :

- Chaque enseignant a évidemment une relation particulière avec ses élèves. En présence d'autres enseignants, les jeux relationnels sont modifiés, pour tout le monde. Le fait même de rechercher davantage de cohérence fragilise le groupe des enseignants : aux yeux des élèves, aux yeux des parents, aux yeux des collègues.

- Les enseignants d'aujourd'hui dont la plupart n'ont pas été baigné dans une culture de coopération, que ce soit en famille, à l'école ou ailleurs. En cas d'échec, il sera plus facile de le vivre avec humour.

- Le bénéfice pour chacun sera profond si la créativité personnelle n'est pas coincée ou facilitée par la pression du groupe et si, au contraire, elle 
s'en trouve stimulée et amplifiée. Surmonter sa jalousie pour arriver à s'émerveiller devant les étincelles d'autrui sans se sentir dévalorisé, créer un climat propice à la pensée divergente, dépourvu d'angoisse.

- L'incompétence d'un ou de plusieurs membres dans leur discipline peut avoir l'un des effets suivants :

- Prédominance de la perspective de l'une des disciplines : des personnes plus compétentes ou sachant mieux articuler leur point de vue vont imposer l'orientation de leur discipline, même si celle-ci n'est pas la plus pertinente.

○ Retrait du projet : les personnes les plus compétentes peuvent ressentir du découragement et adopter une attitude passive.

- Réduction au plus petit dénominateur commun : des erreurs de la plupart des personnes peuvent aboutir à une analyse superficielle, basée sur les informations les plus simples et les plus faciles à comprendre.

En mettant toutes ces obstacles en considération, la chercheuse et les enseignants, échantillon de la recherche, ont déployé tous les efforts pour surmonter ces problèmes, bref les éviter avant même les affronter.

Les avantages de
l'approche interdisciplinaire :
LOWE (2002) affirme que l'interdisciplinarité assure un apprentissage plus réel, concret et global ; et l'apprenant qui subit un projet interdisciplinaire se concentre autant sur « comment il apprend ", sur le développement de sa méthode personnelle de collecte de données et son processus de résolution de problèmes ainsi que sur l'établissement de liens qu'il fait entre les matières que sur les notions apprises pour chaque matière isolée. Elle facilite aussi la création de liens et le transfert de connaissances et d'habiletés entre les disciplines, ce qui rapproche celles-ci de la réalité de la vie de tous les jours. Elle semble avoir pour résultat un meilleur apprentissage global. (Lenoir et Sauvé, 1998) assurent que l'interdisciplinarité permet l'intégration des apprentissages et des savoirs chez les apprenants; de même, (Camel et Fargue Lelievre, 2009) affirment que : "adopter un point de vue interdisciplinaire permet de porter un nouveau regard sur sa discipline ». 
Face à une société en transformation rapide, la formation des enseignants constitue une priorité et fait l'objet de réformes à l'échelle internationale, elle demeure un incontournable allié à l'éducation pour permettre de réunir les conditions suivantes : la réussite et le soutien des apprenants, le développement professionnel des enseignants, et le rendement scolaire; et pour se collaborer pour réaliser cette formation, les pédagogues s'appuyaient sur les valeurs de la citoyenneté numériques qui tend à préparer un citoyen qui sera un individu qui "bénéficie de droits et qui doit s'acquitter de certains devoirs dans une collectivité démocratique ". On peut également y lire « qualité juridique qui garantit à son titulaire la jouissance des libertés publiques et l'électorat 》.

\section{La citovenneté numérique :}

Selon le Grand dictionnaire terminologique, la citoyenneté numérique, c'est d'abord l'ancrage de deux concepts distincts : celui de citoyen et celui de numérique : on dit numérique "une information qui se présente sous forme de nombres associés à une indication de la grandeur à laquelle ils s'appliquent, permettant les calculs, les statistiques, la vérification des modèles mathématiques $»$.
Le terme citoyen numérique est apparu depuis plus d'une dizaine d'années dans le cadre d'un projet intitulé "l'éducation à la citoyenneté numérique ECN 》; mené par la Division des Politiques Éducatives du Conseil de l'Europe, les responsables ont déterminé les objectifs de la citoyenneté numérique dans le fait de permettre aux apprenants de participer activement et de manière autonome à la société numérique.

Ce qui exige une éducation qui développe un sens d'analyse critique et l'utilisation efficace des technologies numériques intégrant une notion de citoyenneté fondée sur le respect des droits de l'homme et la culture démocratique; le conseil de l'Europe 2017 définit la citoyenneté numérique comme étant "Le maniement efficace et positif des technologies numériques et la participation active et responsable aux communautés à tous les niveaux, l'engagement dans un double processus d'apprentissage tout au long de la vie et la défense continue de la dignité humaine. "

Étant donné que le contenu en ligne change de façon permanente, l'éducation à la citoyenneté numérique devrait se concentrer sur les fondamentaux de la citoyenneté et des valeurs humaines afin de permettre un 
enseignement efficace de ce qu'est un comportement responsable en ligne.

Pour pouvoir mieux anticiper ce nouveau concept omniprésent dans notre environnement, on présente cidessous les 10 thématiques concernant l'éducation à la citoyenneté numérique; ils se sont regroupés autour de plusieurs axes :

\section{Axe premier : Être en ligne}

Accès et intégration : C'est l'accès à l'environnement numérique, qui se traduit dans une série de compétences liées à la résolution des différentes formes de fracture numérique et aux aptitudes nécessaires à la participation des futurs citoyens à des espaces numériques ouverts à toutes les opinions diverses. Durant l'application du module, la chercheuse peut affirmer que l'échantillon de la recherche, les 18 enseignants sont dotés de ces compétences.

\section{Apprentissage}

et créativité : C'est le désir d'apprendre et l'attitude que l'on adopte vis-à-vis de l'apprentissage par l'intermédiaire des environnements numériques tout au long de la vie, pour développer et exprimer différentes formes de créativité au moyen de différents outils et dans des contextes multiples et variés. Il couvre les compétences de développement personnel et professionnel qui permettent de se préparer à relever les défis que posent les sociétés à forte composante technologique et à y faire face avec un sentiment d'efficacité personnelle et de manière innovante; la chercheuse a pu sentir la motivation des enseignants sujets de la recherche tout au long de l'application du module.

Maîtrise des médias et de l'information : C'est la capacité à interpréter, et à comprendre en conservant un œil critique et à exprimer la créativité par l'intermédiaire des médias numériques. Un citoyen numérique doit constamment conserver une approche critique s'il veut pouvoir participer pleinement et véritablement à la vie de sa communauté. Nos enseignants ont valorisé l'importance de l'apprentissage/ l'enseignement de ces compétences dans leurs classes.

Deuxième axe : Bien être en ligne :

Éthique et empathie : C'est l'éthique du comportement en ligne et des interactions avec autrui sur Internet et repose sur la capacité à accepter et comprendre les sentiments et les points de vue d'autrui. L'empathie est essentielle à une expérience positive en ligne 
et à l'exploitation des possibilités qu'offre le monde numérique.

Santé et bien-être : Les citoyens numériques se situent dans l'entre-deux des espaces virtuels et des espaces réels : c'est pourquoi l'acquisition de compétences numériques de base ne suffit pas. Les enseignants avec leurs apprenants sont aussi appelés à développer des attitudes, des compétences, des valeurs et des connaissances qui les amènent à devenir plus sensibles aux questions de santé et de bien-être, tels que l'addiction à Internet, l'ergonomie et la position et l'utilisation excessive d'appareils numériques et portables.

\section{Présence}

et

communications en ligne : C'est le développement, chez les citoyens numériques, de qualités personnelles et interpersonnelles qui les aident à conserver une présence et une identité en ligne et des échanges sur Internet qui soient positifs, cohérents et fidèles à ce qu'ils sont.

Il couvre plusieurs compétences, parmi lesquelles la communication et l'échange en ligne via des espaces sociaux virtuels et la gestion de ses données et traces.

Troisième axe : Mes droits en ligne

Participation active en ligne : concerne les compétences que les citoyens doivent posséder pour avoir pleinement conscience des environnements dans lesquels ils évoluent afin de prendre des décisions judicieuses et de participer de manière active et positive aux cultures considérés dans lesquelles ils vivent.

Droits et responsabilités les citoyens numériques du monde en ligne ont certains droits et certaines responsabilités. Ils peuvent exercer, entre autres, leurs droits au respect de la vie privée, à la sécurité, à l'accès et à l'inclusion, ainsi que la liberté d'expression. Mais ces droits s'accompagnent d'un certain nombre de responsabilités, comme l'éthique et l'empathie, ainsi que d'autres visant à garantir un environnement numérique sans risque et responsable pour tous.

Vie privée et sécurité : la vie privée concerne la protection personnelle de ses propres informations en ligne et de celles des autres, tandis que la sécurité est liée à la conscience que l'on a des actes et des comportements en ligne. $\mathrm{Ce}$ domaine concerne diverses compétences comme la bonne gestion des informations personnelles qui sont mises en ligne et de celles des autres ou ce qui a trait à la sécurité en ligne (utilisation de filtres de navigation, de mots de passe, de logiciels antivirus, etc.) pour éviter les 
$\begin{array}{lll}\text { situations dangereuses } \quad \text { ou } & \text { d }\end{array}$ déplaisantes.

\section{Sensibilisation}

des

consommateurs: Le web, avec toutes ses dimensions, notamment les médias sociaux ou d'autres espaces sociaux virtuels, est un environnement dans lequel, souvent, le citoyen numérique est aussi un consommateur. Comprendre les implications de la réalité commerciale propre à beaucoup d'espaces en ligne est l'une des compétences que les individus devront acquérir s'ils veulent pouvoir conserver leur autonomie en tant que citoyens numériques.

A la lueur de ces thématiques, la chercheuse a élaboré une grille des compétences de la citoyenneté numérique nécessaires aux enseignants au service, afin de les transmettre à leurs apprenants dans leurs classes, lors de l'application du module proposé.

Les implications du secteur de l'éducation : En ce qui concerne la préparation il est indispensable de prendre en considération quelques indices des enseignants :

- As du numérique et philosophes : Cela peut prendre la forme d'un effort collaboratif et partagé, où les apprenants sont valorisés comme étant des " as 》du numérique et où les enseignants agissent comme des " philosophes" $d u$ numérique, ou inversement.

- Responsabilité individuelle : du fait des moindres contrôles en ligne, les personnes peuvent s'en sortir plus facilement qu'ils ne le feraient dans la vraie vie. Il est ainsi beaucoup plus facile de faire cause commune avec des activités illégales et de les autoriser à se dérouler en ligne. $\mathrm{La}$ communauté dans son ensemble est directement responsable de l'environnement en ligne et doit lutter contre le « réflexe » qui consiste à laisser les pouvoirs publics et les services répressifs s'occuper des activités illégales.

- Espace d'expérimentation : l'espace en ligne peut être considéré par les jeunes comme un endroit où ils peuvent mener des expériences sous leur identité propre. Les enseignants devraient s'intéresser à l'angoisse que les apprenants peuvent ressentir lors de leurs activités en ligne et encourager une attitude positive à cet égard.

- Une approche plus proactive que réactive : les actions, les politiques et les projets doivent être proposés de manière proactive aux jeunes et à 
l'ensemble des citoyens. Il est facile de faire peur aux gens et de leur enseigner la sécurité en ligne ; cependant, nous devons aller plus loin et montrer les aspects plus reluisants et positifs de la citoyenneté numérique.

- Un processus tout au long de la vie : il est important de considérer l'éducation à la citoyenneté numérique comme un processus qui se déroule tout au long de la vie, qui commence à la naissance et évolue constamment avec le cycle de vie de l'apprenant, c'est exactement ce que cette recherche vise à réaliser par l'intégration des compétences de la citoyenneté numérique dans notre système éducatif.

- Inclusion : les personnes souffrant de handicaps ou ayant des besoins spécifiques doivent être prises en compte afin de veiller à ce que l'éducation à la citoyenneté numérique n'oublie personne.

\section{Les priorités pour avancer vers} la citoyenneté numérique :

Pour réussir à intégrer la citoyenneté numérique dans l'enseignement/apprentissage quelques points de repère sont à prendre en compte

- Communauté : Les éducateurs, les partenaires issus du secteur privé et les autres acteurs concernés, y compris les partenaires sociaux, doivent collaborer en vue $d u$ développement de la citoyenneté numérique.

- Curriculum : Le curriculum trop détaillé, limite la liberté d'improvisation des enseignants. Les curriculums doivent être flexibles et dynamiques, avec des acquis d'apprentissage larges (afin de pouvoir inclure les compétences liées à la citoyenneté numérique)..

- Enseignants : Ils doivent recevoir une formation (avec un accent mis sur les pratiques interactives) car ils ne peuvent pas faire progresser la question seuls. Ils ont besoin d'un enseignement, de ressources et d'un soutien inclusif. Il faut mettre l'accent sur la pédagogie et mettre en avant les interactions entre élèves et entre les élèves et les enseignants, y compris l'interaction interculturelle.

- Ressources : Il faut rendre les ressources validées sur l'éducation à la citoyenneté numérique (par exemple documents imprimés, en ligne, applications) disponibles pour les enseignants et les étudiants. Les enseignants devraient utiliser Internet pour dialoguer 
avec les élèves et leur permettre de traiter des questions en classe. On doit aussi changer la langue du cadre de l'éducation à la citoyenneté numérique en une langue pédagogique, afin que les enseignants puissent faire des liens avec celle-ci.

- Élèves : Ils doivent pouvoir apporter leurs propres expériences du numérique issues de leur vie quotidienne dans la salle de classe afin de pouvoir $y$ réfléchir et de continuer à développer des connaissances et des aptitudes.

Pour conclure on peut affirmer que la formation est essentielle dans le métier des enseignants, vu que ces derniers sont dans un processus d'apprentissage continu afin de répondre aux exigences des apprenants et d'être à la pointe des connaissances. La formation tend à préparer des praticiens capables d'analyser leurs pratiques. Elle tend à sensibiliser les enseignants aux travaux de recherche, à leur procurer des outils issus de cette dernière, de façon à ce qu'ils puissent partager leurs expériences, les interroger, innover et se former collectivement.

\section{L'expérimentation :}

Cette recherche vise à répondre à la question suivante : "comment former les enseignants en service à intégrer les compétences de la citoyenneté numérique à la lueur de l'approche interdisciplinaire ? Pour ce faire, la chercheuse présente dans les lignes à venir le choix de l'échantillon ainsi que les outils de la recherche et les étapes de l'élaboration, l'application et l'évaluation du module proposé.

\section{Échantillon de la recherche :}

En l'année 2018-2019, l'Institut Français, chargé du développement professionnel des enseignants dans les écoles francophones a accordé la responsabilité de former ses enseignants à la chercheuse dans le cadre de la formation continue la deuxième année, pour un module de 20 heures.

Face à cette convocation, la chercheuse a jugé propice de former ces enseignants à l'éducation à la citoyenneté numérique selon l'approche interdisciplinaire. Ainsi, le module proposé était appliqué avec 18 enseignants de français et de DNL (discipline non linguistique) dont les années d'expérience varient entre 5 et 15 ans; ils enseignent selon différents systèmes éducatifs : égyptiens, français, anglais et américains.

\section{Outils de la recherche :}


1- Le questionnaire des compétences numériques : ${ }^{1}$

But : Déterminer les compétences numériques nécessaires pour maîtriser et acquérir les compétences de la citoyenneté numérique des enseignants.

Source : en se basant sur le référentiel TIC de l'UNESCO préparé par le conseil de l'Europe, ${ }^{2}$; la chercheuse a sélectionné parmi les compétences de la numérique citées, celles qui conviennent le mieux avec l'environnement et le niveau de notre système éducatif.

\section{Description :}

ce

questionnaire se compose de 45 compétences réparties en trois axes.

Le premier axe réside dans les activités de production et de gestion qui englobent: la recherche et le traitement de l'information, la production des matériels didactiques tels que les notes de cours, les cahiers d'exercices et le logiciel de traitement de texte; la communication électronique avec les collègues tels que les courriels, les forums, le WhatsApp; et la gestion des

${ }^{1}$ Annexe no :1 résultats des étudiants selon les logiciels appropriés.

Les enseignants en service sont dotés de la plupart de ces compétences ; ils les pratiquent spontanément durant leurs préparations hebdomadaires.

Le deuxième axe intitulé les activités de diffusion multimédia se compose des sous compétences, telles que: la présentation des multimédia, la sélection, le traitement de la planification et la diffusion de toutes les ressources tels que les images, les sons, les films; et tout autre contenu approprié aux objectifs technopédagogiques des cursus enseignés.

La plupart des enseignants ont eu recours à ces activités selon leur discipline, mais certaines difficultés peuvent en dépasser la réalisation, telle que la connexion faible de l'Internet dans leurs établissements surtout avec les ressources qui exigent un fort accès à l'Internet.

Le troisième axe concerne les activités d'apprentissage interactif telles que la réalisation d'un site web avec une application par les étudiants, ainsi que l'utilisation parfaite des outils de communication ayant pour but de favoriser l'apprentissage collaboratif et la construction collective des connaissances tout en mettant en considération les 
activités d'évaluation formative et sommative.

Ces compétences étaient les moins réalisées par les enseignants, personne n'a essayé de créer un site web ou un blog; ils se contentent d'avoir accès aux plateformes de leurs écoles, ils ont recours aux activités collectives des connaissances par le biais des projets menés par leurs établissements.

\section{2- La grille des compétences de} la citoyenneté numérique ${ }^{1}$ :

But : cette grille vise à déterminer les compétences de la citoyenneté numérique.

Sources et élaboration : Pour élaborer cette grille, la chercheuse a suivi les étapes suivantes :

- Analyser les études concernant l'éducation à la citoyenneté numérique.

- Sélectionner les compétences qui convient avec le niveau linguistique, professionnel et numérique des 18 enseignants de français en service, échantillon de la recherche.

- Formuler les compétences dans leur forme finale.

Description : cette grille se compose de 25 compétences réparties en trois axes essentiels à savoir: Être en ligne: qui

${ }^{1}$ Annexe no : 2 comporte l'échange des informations correctes, axe $\mathbf{2}$ : Bien être en ligne qui englobe l'étiquette numérique; et axe 3 Mes droits en ligne concernant les compétences du respect de la loi et la protection de la santé ; valorisant les savoir-faire numériques, les attitudes et les valeurs relatifs à la citoyenneté numérique. Une certaine progression des sous compétences est prise en considération dans l'élaboration de la liste.

L'application de cette grille mène la chercheuse à interpréter quelques faits :

- La citoyenneté numérique est une expression nouvelle pour eux.

- $(45 \%)$ de l'échantillon essayent d'appliquer l'étiquette numérique.

- $(60 \%)$ des enseignants en service suivent des comportements erronés concernant les échanges des informations.

- $(70 \%)$ des enseignants n'ont aucune idée de leurs droits ou de leurs devoirs qui régissent l'utilisation numérique.

- $(90 \%)$ des enseignants souffrent des maladies de l'utilisation excessif du numérique, telle que (Syndrome du canal carpien, fatigue des yeux, mauvaise 
position, addiction à Internet, syndrome de stress répétitif.

- $(100 \%)$ de l'échantillon évitent de faire des achats en ligne, par prudence et aussi par manque de confiance et le risque d'être victime de tomber dans des tentations de fraude et d'escroquerie.

\section{3- Le pré/post-test ${ }^{1}$ :}

Buts : Pour répondre à la deuxième question de la recherche : Jusqu'à quel point les enseignants en service sont dotés des compétences de l'intégration de la citoyenneté numérique selon l'approche interdisciplinaire? La chercheuse a élaboré ce pré test avant la mise en application du module proposé.

Le même test sera postérieurement appliqué après l'application du module proposé afin de mesurer l'efficacité du module pour la formation des enseignants à intégrer les compétences de la citoyenneté numérique selon l'approche interdisciplinaire.

Description : Le pré/ post-test se compose de 4 questions à réponses ouvertes qui mesurent les connaissances et les savoirs, les attitudes et les valeurs relatifs au sujet de l'éducation à la citoyenneté numérique, et le profil d'un bon enseignant numérique responsable. On compte 20 points pour cette partie.

Une cinquième question porte sur la conception pédagogique à acquérir, l'évaluation de savoir-faire didactique, elle a pour but de mesurer les performances des enseignants à intégrer les compétences de la citoyenneté numérique selon l'approche interdisciplinaire, on compte 20 points pour cette partie.

Le calcul du temps : Étant donné que le pré/ post-test est composé de deux parties, on a jugé nécessaire de consacrer aux questions appartenant à chaque partie un temps fixe pour $y$ répondre.

Une pré-application du test sur un groupe d'enseignant à l'Institut Français, permet de déterminer le temps moyen qui était calculé selon cette formule.

Temps mis par le premier enseignant + temps remis par le dernier enseignant divisé par 2 :

$\frac{10+30}{2}=20$ minutes pour la première partie. $\frac{20+60}{2}=40$ minutes pour la deuxième partie.

-Ainsi le temps du test dans sa totalité est $\mathbf{6 0}$ minutes (une heure)

${ }^{1}$ Annexe no : 4 
Fiabilité: Pour calculer la fiabilité du pré post /test, nous avons eu recours à l'équation d'Alpha selon laquelle la fiabilité fut prouvée au niveau de 0.87 .

Validité: La validité du pré post test fut de même calculé à partir de sa fiabilité :

$$
\mathrm{V}=\sqrt{0.87}=0.93
$$

\section{4- Le module proposé ${ }^{1}$}

La description du module
proposé: Le module est
organisé sous forme de 10
séances.
Séance 1 : l'application du pré
test et du questionnaire
des compétences
numériques et celles de la
citoyenneté numérique;
tout en introduisant aux
stagiaires le module
proposé.

Séance 2: les composantes de la citoyenneté numérique et les conseils à concevoir pour être un bon citoyen numérique.

Séance 3: les responsabilités du citoyen numérique: ses droits et ses devoirs.

Séance 4: les valeurs de la citoyenneté numérique et les attitudes censées

\footnotetext{
${ }^{1}$ Voir le module en détails annexe no 3
}

promouvoir chez les apprenants.

Séance 5: l'interdisciplinarité dans la pratique pédagogique et ce qui distingue les termes de multi, pluri, inter et transdisciplinarité ; et les étapes à suivre pour enseigner selon cette approche.

Séance 6: discussion et présentation d'une leçon modèle de la citoyenneté numérique.

Séance 7- 8 et 9 : les stagiaires présentent leurs travails de groupe en fonction de 2 groupes par semaine, le totale est 6 leçons de 6 groupes.

Séance 10 : l'application du post test et la fiche d'autoévaluation et la discussion $\mathrm{du}$ portfolio professionnel.

Chaque séance vise à réaliser un objectif final, décomposé en objectifs spécifiques jugés essentiels à la réalisation de l'objectif final du module proposé : l'intégration de la citoyenneté numérique. selon l'approche interdisciplinaire.

Déroulement de la formation du module :

Avant l'application du module proposé on présente aux 
enseignants les principes à respecter pour le travail en équipe interdisciplinaire. Pour travailler en équipe interdisciplinaire, Sellamna, N. (2014) propose de respecter les principes suivants :

- S'accorder sur des objectifs partagés.

-Aborder le travail interdisciplinaire sous l'angle d'un problème à traiter l'éducation à la citoyenneté numérique plutôt que d'une méthode à mettre en œuvre.

- Identifier rapidement les contributions de chaque discipline, (le français, les mathématiques et les SVT) selon le cas des stagiaires, les compétences disponibles, programmer et distribuer les tâches.

- S'entendre sur le fait qu'il n'y pas de résultats qualitatifs, si les participants ne sont pas pleinement compétents dans leur propre domaine.

- S'intéresser et comprendre les systèmes de valeurs des disciplines associées : comprendre et apprécier les apports de chaque discipline et leur contribution, comprendre le vocabulaire et les concepts spécifiques à chaque domaine pour communiquer entre disciplines, percevoir les similarités et les différences entres les disciplines.
On leur présente aussi comme indications générales pour le travail en équipe et individuel :

Mieux

fait

individuellement : Rassembler les informations, élaborer les idées, apporter des propositions, préparer les sessions de travail d'équipe et formuler et écrire les textes.

Mieux fait en équipe : Produire les idées, échanger les expériences, communiquer les informations et prendre les décisions.

Une erreur sur l'un ou plusieurs des aspects du sujet à traiter peut fausser les jugements de toute l'équipe. De même, une erreur dans la maîtrise d'une méthode inhérente à une discipline, amoindrit la capacité de l'équipe à rassembler des informations pertinentes et valides.

La séance avec laquelle on débute la conception pédagogique (séance 6) avait pour objectif que les stagiaires se retrouvent en groupes hétérogène, inclus les enseignants de (français, math, et $\mathrm{SVT}^{1}$ ), après un tour de table visant à permettre à chacun d'annoncer la discipline qu'il dispense, chaque membre du groupe décrit en quoi lui consiste la discipline mentionnée, à ce moment tous les clichés liés aux

${ }^{1}$ Science de la vie et de la terre 
différentes disciplines sortent, et les stagiaires commencent à regrouper les compétences afin de formuler les objectifs sur les compétences transversales semblables afin de travailler les mêmes savoirs mais sous angles différents; ce travail en petits groupes est organisé de façon telle que chacun ait sa part de responsabilité et que chacun soit indispensable pour mener à bien son travail ; et dans deux semaines, ces groupes commencent à défendre chacun leur présentation devant leurs pairs, cette procédure dure pour ( 3 séances en raison de 2 groupes par séance ).

L'évaluation du module se fait au moyen d'une grille d'observation des stagiaires ${ }^{1}$; une fiche d'auto-évaluation ${ }^{2}$ et le post test ${ }^{3}$. Les résultats des évaluations montrent que les stagiaires ont apprécié le travail interdisciplinaire surtout en fréquentant un nouveau domaine tel que la citoyenneté numérique.

Lieu et temps, et équipement de l'application du module :

Ce module a eu lieu chaque jeudi de $9 \mathrm{~h}$ : à $11 \mathrm{~h}$ dans l'école des frères Bab Ellouq, pendant 10 jours à raison de 2 heures par jour,

1 Annexe no :6

${ }^{2}$ Annexe no:7

${ }^{3}$ Annexe no : 3 l'application de cette recherche a eu lieu dans une salle climatisée, équipé par un ordinateur, data show, grand écran, avec une capacité de 25 stagiaires au maximum; les stagiaires ont travaillé pour la plupart du temps sur leur ordinateurs portables afin de faire le travail dans une salle semblable à celle de leurs écoles.

\section{5- La fiche de préparation de la lecon $^{4}$}

But: Cette fiche offre des pistes d'exploitation aux enseignants pour mettre en valeur une expérience de préparation d'une leçon selon l'approche interdisciplinaire .

\section{Descriptif :}

Chaque enseignant détermine les concepts de sa discipline en relation avec la citoyenneté numérique, puis ils les regroupent afin de formuler les objectifs spécifiques.

Ils déterminent un contenu qui s'adapte avec leurs disciplines, par suite ils précisent les activités à réaliser, et les modules de travail. La démarche en trois temps que nous proposons: sensibilisation, structuration et réemploi permet aux stagiaires à partir de leurs expériences, d'interagir, de comprendre, d'interpréter, et d'apprécier les concepts et les activités concernant la citoyenneté

${ }^{4}$ Annexe no : 5 
numérique, chacun selon sa discipline, ainsi, les activités proposées peuvent s'échelonner sur plusieurs cours, voire plusieurs semaines.

\section{6- La grille d'observation :1}

But: afin d'évaluer les compétences de l'éducation à la citoyenneté numérique, la chercheuse a formulé une grille d'observation en vue d'évaluer les performances des stagiaires lors de la préparation d'une leçon concernant la citoyenneté numérique selon l'approche interdisciplinarité.

Description : elle se compose de 20 performances que les stagiaires doivent réaliser lors de la phase de la conception pédagogique. Ces performances sont réparties sur 3 axes principaux, la préparation du travail, l'exécution et la réalisation; et l'évaluation. Ces performances varient entre la formulation et le lancement des objectifs, le choix du contenu, des stratégies et du modalités de travail adaptés aux objectifs censés réalisés ainsi que le niveau des apprenants.

Ces performances recouvrent aussi la variation des activités proposés et leurs adéquations aux objectifs et au contenu, tout en mettant en considération l'interaction avec toutes ses sortes, quand même l'utilisation efficace de la technologie et la variation des supports aussi que le fait de s'assurer de la réalisation de l'objectif destiné par le biais de l'évaluation durant et à la fin de la séance préparée.

\section{Critères de notation :}

Chaque performance est notée d'un point si l'enseignant l'a bien appliquée et du Zéro si non :

Chaque enseignant doit réaliser au moins $80 \%$ de ces performances, sinon il doit refaire le travail et la présentation; pour réussir la note doit dépasser $16 / 20$ points; Il est à noter que les 18 enseignants ont dépassé le taux de $80 \%$, ce qui est due à leurs expériences qui varient entre 5 et 24 ans de travail comme enseignant avec divers niveau des apprenants, mais ce qui était de nouveau pour eux, était le thème traité et le travail interdisciplinaire qui a suscité la curiosité et le plaisir durant le travail selon leur commentaire dans le portfolio professionnel.

\section{7- La fiche d'auto-évaluation des compétences de la $\mathrm{CN}^{2}$}

But : cette fiche tend à aider chaque stagiaire à s'évaluer: ses

2 Annexe no 7 
compétences, ses valeurs et les attitudes acquises tout au long de la pratique du module proposé.

La chercheuse a aussi encouragé les enseignants à appliquer à leur tour avec leurs apprenants l'ECN dans leurs institutions.

Description : cette fiche met en valeur les composantes de la citoyenneté numérique citées par le Conseil d'Europe, elle se compose de 5 axes principaux :

L'étiquette de la numérique dans le sens de gérer les relations virtuelles en ligne comme: la réduction du sarcasme, le fait d'accepter les autres et de les respecter.

L'axe concernant l'échange des informations correctes qui soutient la prise de décision correcte avant la publication de n'importe quelle information soit personnelle ou générale et le fait d'être prudent en ce qui concerne les contacts en ligne.

L'axe troisième traite le respect de la loi, et la conscience par les droits et les devoirs qui règlent les comportements en ligne, les politiques du copyright et la protection des ressources et des médias.

Le quatrième axe concerne la protection de la santé et le fait de protéger son corps de toutes les positions erronées causant tant de maladies.

L'axe dernier qui concerne le commerce numérique avec toutes les précautions de l'achat et les transactions bancaire.

En ce qui concerne les critères de notation, l'enseignant doit noter le degré d'acquisition de chaque item, et pour juger ses performances, il doit calculer les notes ainsi: un point s'il ne possède $\mathrm{du}$ tout la compétence (jamais), 2 points s'il l'applique (parfois) et 3 points s'il accomplit (toujours) cette performance.

L'analyse des résultats : un stagiaire parmi les 18 n'était jamais un citoyen numérique, il a obtenu moins que 35 , et aussi un autre était excellent, ses notes ont dépassé le 90, de même 7 stagiaires étaient sur le point d'être des bons citoyens numériques, et 9 parmi eux ont été jugé comme de bons citoyens, ce qui assure l'efficacité du module proposé sur le développement des compétences de la citoyenneté numérique de nos stagiaires.

\section{8- Le portfolio professionnel :}

But : cet outil a pour but de planifier et prévoir les apprentissages des enseignants en cours de service lors de l'application du module proposé, il

1 Annexe no 8 
est destiné à recueillir les données concernant les attitudes et les valeurs de la citoyenneté numérique traités lors la préparation de leur travail interdisciplinaire; ce portfolio professionnel accompagne l'analyse des situations professionnelles surtout en ce qui concerne la mise en commun des objectifs et des concepts des 3 disciplines traités (français, math et science). Il sert comme un "brouillon" qui oriente leurs choix, encadre les contenus concernant la citoyenneté numérique et l'interdisciplinarité en étant la méthode retenue, il alimente les moments de soutien et de remédiation par les contenus qui répondent aux vrais besoins de leurs apprenants; il favorise les prises de décisions en matière de soutien motivées, efficaces et pertinentes. Tout au long de l'application du module proposé, ils notent leurs commentaires, ils détectent les difficultés rencontrées et les discutent pour les surmonter, ce qui les aident à avoir une vision claire et exacte sur les difficultés de leurs classes, et leurs permettront de mener une autoréflexion bien fondée sur leurs actions pédagogiques.

En ce qui concerne l'expérimentation :

- Certains enseignants soulignent leur manque de familiarité avec le concept d'interdisciplinarité : «Avant de commencer ce stage, je n'avais jamais entendu ce mot $\gg$.

- Tous ont du mal à observer des situations de classe interdisciplinaires: " quand j'ai commencé mon stage, je me suis d'abord rendu compte qu'il n'y avait pas d'interdisciplinarité. "

- De même les réticences à l'égard de l'interdisciplinarité semblent réelles : "Une belle idée sur le papier, mais personne ne le fait " explique l'un d'entre eux.

- Il y a parmi ces stagiaires qui ont soutenu l'idée que : "tout devrait être développé en lien avec les thématiques tirées de la vie quotidienne et qu'une approche plus interdisciplinaire devrait être plus systématiques appliquée "

- Un des enseignants se place parmi les opposants de l'interdisciplinarité : "elle n'est qu'un moyen et elle ne doit pas être vue comme une fin en soi " ces collègues ont bien respecté son point de vue.

- Les enseignants se sont trouvés dans l'obligation de rechercher dans les sites web 
des contenus traitant ce nouveau domaine, "les redondances des supports en ligne proposée par Le Conseil de l'Europe m'ont donné l'impression que nous sommes très en retard en ce qui concerne l'ECN"

- Le domaine de la sécurité des apprenants et leur protection dans l'environnement numérique, plutôt que leur responsabilisation et autonomisation, était le thème le plus traité lors de la préparation de leur travail en commun, grâce à l'importance de l'acquisition de ces compétences pour participer activement à la société numérique.

- Les rencontres accordées par chaque groupe pour mettre en commun les objectifs et les concepts de chaque discipline ont suscité des discussions fructueuses et créatives, "Ces rencontres nous ont encouragés à partager nos expériences" ce qui les mènent à faire plus de recherche sur le thème abordé.

- Ils ont apprécies la fiche d'auto-évaluation; ils ont pu déterminer leurs niveaux visà-vis les compétences de la citoyenneté numérique et ils ont demandé de l'appliquer sur leurs apprenants lors de l'éducation à la citoyenneté numérique .

- Les enseignants qui ont participé à cette recherche ont d'ailleurs éprouvé un certain intérêt à élaborer un projet interdisciplinaire; cette expérience les a permis de se familiariser aux savoirs qui fondent l'identité professionnelle, elle les aide aussi à se détacher de leur discipline académique et à mieux appréhender les contours et la spécificité de leur discipline d'enseignement sur le plan des contenus et des méthodes, ils ont eu l'occasion aussi de porter un regard neuf sur leur propre discipline, et à porter un regard critique sur les plans d'études.

\section{Analyse qualitative quantitative de la chercheuse :}

Au début de l'application du module, les enseignants étaient déçus, non-enthousiasmés, pressés par le travail, les cours et les examens. Après la présentation d'un aperçu général des objectifs du module, ils commencent à sentir un intérêt vis-à-vis de sujet proposé.

Le remue-méninge qui débute toujours chaque séance a fourni une atmosphère d'amicalité et d'enthousiasme entre les enseignants, de même, le travail 
collaboratif les rend encouragés, les aide à échanger les connaissances et les expériences. Les sujets des documents (vidéo, audio, et écrit) ont été d'un grand intérêt pour eux. A la fin de l'expérimentation, les enseignants ont confiance en leur capacité d'enseigner la citoyenneté numérique, ils se mettent d'accord sur leurs besoins de développer leurs compétences concernant l'éducation numérique.

Un nouveau concept digne à être acquérir et à travailler pour se préparer à l'ère numérique. La chercheuse a senti la satisfaction des stagiaires sur le contenu et l'application du module (si on ose le dire), la plupart des stagiaires ont demandé d'assister à d'autres stages dont l'objectif est de développer aussi leurs compétences professionnelles, et technologiques. Bref, le module a

\section{bien intéressé les stagiaires. \\ Tableau $n: 1$ le pré-post test (le tout): (40pts)}

\begin{tabular}{|c|c|c|c|c|c|c|c|c|}
\hline T .des notes & N & Min & Max & $\mathbf{M}_{\mathbf{A}}$ & $\mathbf{E}$ & $\mathbf{D L}$ & $\mathbf{T}$ & $\mathbf{S}$ \\
\hline Pré-test & 18 & 4 & 16 & 10 & 4 & \multirow{2}{*}{17} & \multirow{2}{*}{42} & $\begin{array}{c}\text { Au niveau } \\
\text { de } 0.001\end{array}$ \\
\hline Post-test & 18 & 26 & 36 & 31 & 3.2 & & & \\
\hline
\end{tabular}

Commentaire: Les notes du pré-test (le tout) sont reparties entre 4 et 16 tandis que celles du post test sont reparties entre $\mathbf{2 6}$ et 36 . Ce qui met en relief le développement des enseignants à l'égard du module proposé, de même, la valeur de « $\mathbf{T}$ » est
Pour conclure on peut avouer que mettre en œuvre des pratiques interdisciplinaires avec un thème nouveau par des professeurs peu familiarisés avec l'interdisciplinarité était un défi ; ce qui était surmonté par l'utilisation des outils d'accompagnement aidant à l'acquisition de compétences professionnelles, et même si les avis divergent à ce propos, tous les spécialistes s'accordent sur le fait que pour mettre en place ces projets interdisciplinaires, des bases disciplinaires solides s'avèrent indispensables.

\section{Analyse des résultats}

Chaque tableau indique le minimum et le maximum des notes obtenues par les enseignants dans le pré post test: "le tout et les deux parties du test », la moyenne arithmétique des notes, l'écart type, les degrés de liberté et la valeur de $« \mathrm{~T} »$ au niveau de 0.001 .

significative au niveau de $\mathbf{0 . 0 0 1}$ ce qui met en valeur la progression dû à l'application du module dans sa note totale sur le niveau des enseignants, ainsi la première hypothèse de la recherche est réalisée. 
Tableau n :2 le pré-post test (la partie théorique): (20pts)

\begin{tabular}{|c|c|c|c|c|c|c|c|c|}
\hline \hline T des notes & $\mathbf{N}$ & Min & Max & $\mathbf{M}_{\mathbf{A}}$ & $\mathbf{E}$ & $\mathbf{D L}$ & $\mathbf{T}$ & $\mathbf{S}$ \\
\hline Pré-test & 18 & 0 & 6 & 3 & 2.1 & \multirow{2}{*}{17} & \multirow{2}{*}{30} & $\begin{array}{c}\text { Au niveau } \\
\text { de } 0.001\end{array}$ \\
\hline Post-test & 18 & 10 & 18 & 14 & 2.2 & & & \\
\hline
\end{tabular}

Commentaire: Les notes du pré-test (la partie théorique) sont reparties entre $\mathbf{0}$ et 6 tandis que celles du post test sont reparties entre 10 et $\mathbf{1 8}$. Ce qui met en relief le développement des enseignants à l'égard du module proposé, de même, la valeur de

« $\mathbf{T}$ » est significative au niveau de $\mathbf{0 . 0 0 1}$ ce qui met en valeur la progression dû à l'application du module en ce qui concerne la partie théorique sur le niveau des enseignants, ainsi la deuxième hypothèse de la recherche est réalisée

Tableau $\mathrm{n}: 3$ le prépost test (la conception pédagogique): (20pts)

\begin{tabular}{|c|c|c|c|c|c|c|c|c|}
\hline \hline T. des notes & $\mathbf{N}$ & Min & Max & $\mathbf{M}_{\mathbf{A}}$ & $\mathbf{E}$ & $\mathbf{D L}$ & $\mathbf{T}$ & $\mathbf{S}$ \\
\hline Pré-test & 18 & 4 & 12 & 8 & 2,4 & \multirow{2}{*}{17} & 41.3 & $\begin{array}{c}\text { Au niveau de } \\
0.001\end{array}$ \\
\hline Post-test & 18 & 26 & 18 & 16 & 1.6 & & & \\
\hline
\end{tabular}

Commentaire : Les notes du pré-test sont reparties entre 4 et 12 tandis que celles du post test sont reparties entre 14 et $\mathbf{1 8}$. Ce qui met en relief le développement des enseignants à l'égard du module proposé, de même, la valeur de « $\mathbf{T}$ » est significative au niveau de 0.001 ce qui met en valeur la progression dû à l'application du module en ce qui concerne la conception pédagogique sur le niveau des enseignants, ainsi la dernière hypothèse de la recherche est réalisée

Finalement on peut avouer que les enseignants doivent servir de modèles à leurs élèves. Ils doivent être à la fois des as du numérique et des philosophes : des philosophes afin de comprendre l'importance du concept de la compétence numérique en ligne et hors ligne, et des as du numérique en raison de la rapidité des innovations dans ce domaine. Pour donner aux enseignants les moyens d'allier efficacement compétences techniques et compréhension de la nécessité de la citoyenneté numérique comme outil essentiel pour la dignité humaine, l'une des solutions consiste à leur donner plus d'autonomie. Les résultats de l'expérience à prouver l'efficacité du module proposé sur le développement des compétences de l'enseignement de la citoyenneté numérique en plus de son acquisition. 
Résultats du pré /post test

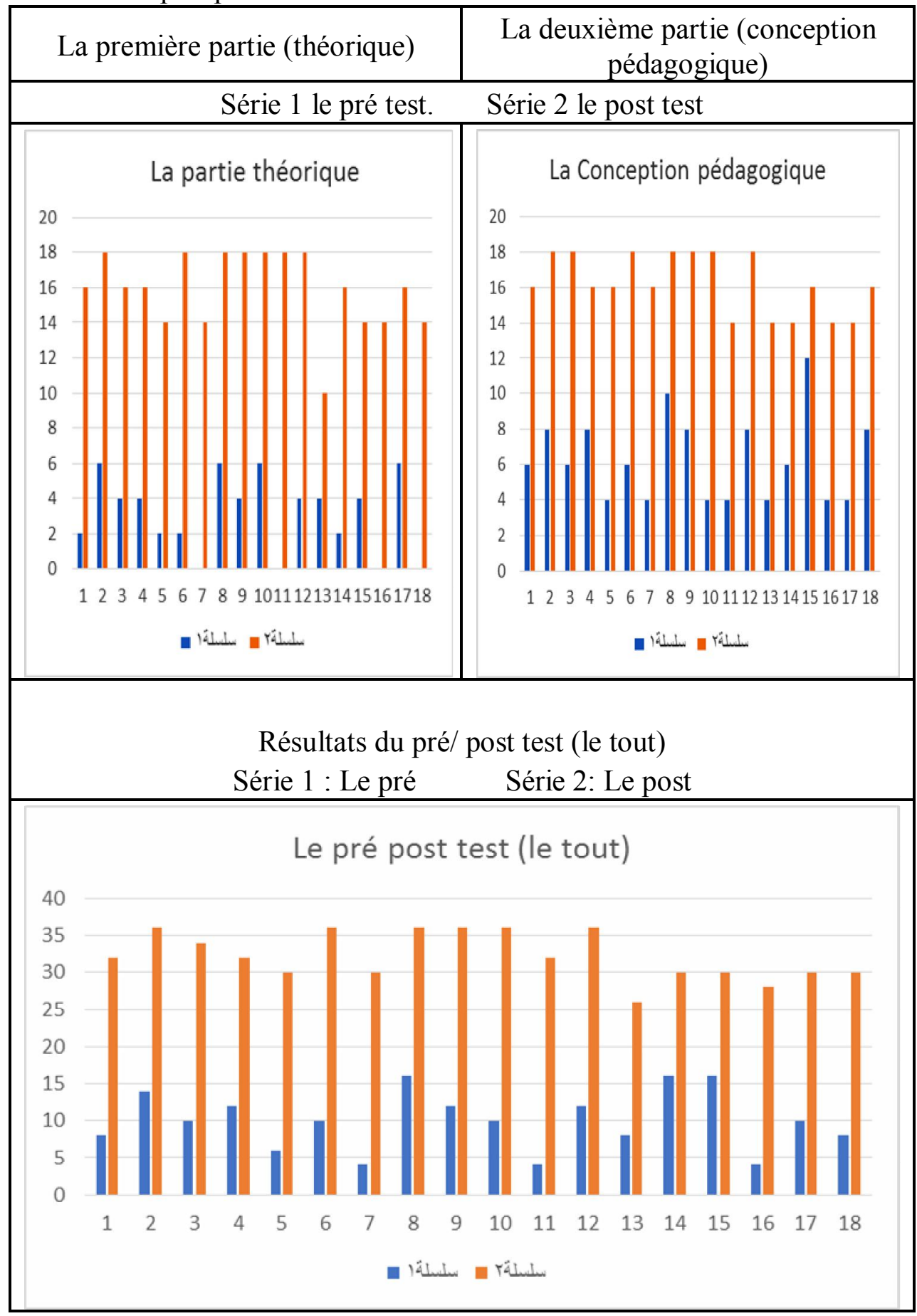




\section{Quelques recommandations :}

A la lueur des résultats, la chercheuse présente ce qui suit :

- Les enseignants doivent pouvoir acquérir des compétences numériques lors de leur formation initiale et continue.

- La formation des enseignants devrait plutôt se concentrer sur la pédagogie, en soulignant les interactions d'élève à élève et d'enseignant à élève, et faire appel aux compétences et aux aptitudes de la citoyenneté numérique.

- Les chefs d'établissement vont être confrontés à des défis majeurs $\mathrm{du}$ fait $\mathrm{de}$ l'environnement numérique et auront donc besoin de formations afin de pouvoir être en pointe de l'apprentissage de l'éducation à la citoyenneté numérique.

- Les preneurs de décisions doivent prendre conscience de l'importance de l'éducation à la citoyenneté numérique et de dégager les budgets nécessaires afin de veiller à ce qu'un effort durable soit fait.

- Il est essentiel de mettre l'accent sur le secteur de la petite enfance et d'envisager l'éducation formelle, informelle et non formelle comme un tout, notamment en gardant à l'esprit que certaines compétences du cadre de la citoyenneté ne seront jamais acquises si elles ne le sont pas pendant les premières années.

- Les enfants utilisent les technologies numériques à un âge précoce et que les parents ne sont pas très au fait des questions de sécurité et de citoyenneté numérique (certains parents utilisent les technologies numériques comme une « baby-sitter »). Le Réseau Social Européen suggère de former les professionnels de la petite enfance afin qu'ils puissent travailler et former les parents à leur tour.

- On doit également former les autres professionnels qui travaillent avec les parents et les élèves.

- Il est important d'associer les enseignants en tant que chercheurs pour favoriser les communautés de pratique.

- On doit aussi intégrer la technologie à ces plateformes (l'utilisation de webinaires) et leur donner forme au sein d'un cadre pertinent, afin de permettre un partage effectif des pratiques d'éducation à la citoyenneté numérique. 
- Les enseignants devraient avoir l'expérience et la formation nécessaires pour utiliser les technologies et réfléchir aux questions de citoyenneté numérique.

- Il est nécessaire d'élaborer des ressources et des documents d'apprentissage. Les MOOC et les salles de classe virtuelles peuvent aussi être de bonnes solutions pour développer l'éducation à la citoyenneté numérique.

- Il convient d'élaborer des lignes directrices à l'intention des décideurs afin d'expliquer pourquoi la citoyenneté numérique est importante.

- L'évaluation doit être partie intégrante de l'approche de la citoyenneté numérique dans les curriculums. On a besoin de différents types d'outils d'évaluation pour les différentes fonctions de l'éducation à la citoyenneté numérique (par exemple les portfolios peuvent être évalués par les pairs, les parents, etc., pour encourager l'autoévaluation).

- Il manque également une série de méthodes pédagogiques. On a besoin de ressources pour des approches qui puissent être mises en œuvre dans différents contextes culturels et linguistiques. Cet outil pourrait être interactif afin que les enseignants puissent ajouter des détails de leur contexte sur le site et obtenir des conseils pertinents

\section{Bibliographie :}

- ALPE,Y. (2006). Savoirs savants et disciplines scolaires : Peut-on enseigner des « sciences sociales "? Communication proposée au colloque de Clermont Ferrand.

- ASTOLFI, J-P. (2006). Les questions vives en question ? In A. Legardez \& L. Simonneaux. L'école à l'épreuve de l'actualité. Enseigner les questions vives. Paris

- ASTOLFI, J-P. (2008). La saveur des savoirs. Paris

- AUDIGIER, F. (2006,). L'interdisciplinarité à l'école Quelques interrogations théoriques et pratiques à propos de l'histoire, de la géographie et de l'éducation citoyenne. http://www.jsse.org/20062/audigier interdisciplinarity frz .htm

- BACHELARD, G. La formation de l'esprit scientifique. Paris : Librairie philosophique, J. Vrin, 1967. ED-86/WS/78 - page 62.

- BAILLY J.-M., et SCHILS J., (1988). Trois niveaux d'interdisciplinarité dans l'enseignement secondaire, 
FNEC, journée pédagogique, Bruxelles.

- BARRERE, A. (2006). Travail scolaire, travail enseignant. In J. Beillerot \& N. Mosconi (dir.). Traité des sciences et des pratiques de l'éducation. (pp.395-405). Paris.

- CHASSOT, I. (2003). La Déclaration de la CIIP relative aux finalités et objectifs de l'Ecole publique, du 30 janvier 2003. Conférence de presse.

- COLLINOT, A. \& MAZIERE F. (1999). Le français à l'école, un enjeu historique et politique. Hatier : Paris.

- Conseil de l'Europe : Le Rapport général ED/DCE (2017) 08 2122 septembre 2017 Strasbourg

- DE KESEL M.:Pourquoi et comment former les futurs enseignants l'interdisciplinarité? (UCL)

- GIORDAN A. et SOUCHON C., (1992). Une éducation pour l'environnement, Z'Editions, Paris.

\section{- LANNOYE}

C., Interdisciplinarité à l'école, dans Apprendre la démocratie et la vivre à l'école, Labor, Bruxelles, pp 59-62.

- LUMBROSO M., (2017) : Le rôle des enseignants à l'ère numérique journées et colloauesm rédiger des comptes rendus Paris.

- MADEL, J. (2014). Former les enseignants à l'ère du numérique : cas d'Haïti. Québec : Livres en ligne du CRIRES. En ligne http://lel.crires.ulaval.ca/public/n umerique haiti

- MATTHEW, J. (2012), Directeur de la Citoyenneté démocratique et de la Participation au Conseil de l'Europe,

- MORIN E., 1977, La méthode, 1. La Nature de la Nature, Paris, Le Seuil.

- PARTOUNE C., 1996, Interdisciplinarité, dans Les Jeunes et la ville - Cadres de références, Communauté française de Belgique, Bruxelles, pp 9-16.

- PERRENOUD P., 1996, Enseigner : agir dans l'urgence, décider dans l'incertitude, chapitre 5 : Travailler en équipe pédagogique, résistances et enjeux.

- PERRENOUD Ph. (2011)Quand l'école prétend préparer à la vie... Développer des compétences ou enseigner d'autres savoirs? Issy-lesMoulineaux : ESF, 2011, 221 p.

- REVERDY C., (2016)
L'utilisation
l'interdisciplinarité dans le
secondaire Veille et analyse 
L'essentiel sur IFé Ens Janvier ; Lyon

- REVERDY, C. , (2015) « Éduquer au-delà des frontières disciplinaires $\square$ », Dossier de veille de l'IFÉ, $\mathrm{n}^{\circ} \cdot 100$, ENS de Lyon, Mars

- SELLAMNA N., (2014 ) : L'interdisciplinarité Ressources Pédagogiques ICRA (www.icraedu.org)

- SOBHY Sh., et MOHAMED E , (2014) Critères de l'éducation à la citoyenneté numériques et ses applications dans les cursus scolaires. Université Almonoufiya

- TIC UNESCO: un référentiel de compétences pour les enseignants; 2012

- VOSSKAMP W., (1983) Colloque de Bucarest "Interdisciplinarity in Higher Education". Bucarest CEPES/Unesco, p. 24 et 25.

\section{Sitographie :}

- http://www.interdisciplines.org/i ndex.cfm

- www.coe.int/dce

- http://www.crdp.acgrenoble.fr/tpe/selecdoc/methodo /inter.htm

- http://perso.clubinternet.fr/nicol /ciret/ bulletin /b2c2.htm
- http://www.is.wayne.edu/mnissa ni/pagepub/smoothie.htm

- http://www.chez.com/brunofortin /sme 1020/

- http://parcoursdiversifies.scola.acparis.fr/parcourssciences/interdis ciplinarite6.htm

- http://www.tms.com.au/tms122c.html

- https://rm.coe.int/educationcitoyennete-numeriqueconference-travail-2017-rapportgenera/16808dd9d6

- https://www.cforp.ca/tactic/resso urces/fiches-dactivites-surlidentite-et-citoyennetenumerique/

- http://www.aixmrs.iufm.fr/forma tions/filieres/ses/didactique/savsa v.html http://www.ciip.ch/pages/presse/ Dos de pre/fichiers/Allocution Chassot.pdf

- http://www.edudoc.ch/static/web /aktuell/medienmitt/eva_f.pdf

- https://citoyennetenumeriqueque bec.ca/eduquer/

- https://www.youtube.com/user/a pprendreetguider 